\title{
Globe
}

Revue internationale d'études québécoises

\section{Des réseaux en convergence. Les espaces de la sociabilité littéraire au féminin dans la première moitié du $\operatorname{XIX}^{\mathbf{e}}$ siècle Converging Networks. Women's Literary Sociabilities in $19^{\text {th }}$ Century Québec}

\section{Julie Roy}

Volume 7, numéro 1, 2004

Réseaux et identités sociales

URI : https://id.erudit.org/iderudit/1000831ar

DOI : https://doi.org/10.7202/1000831ar

Aller au sommaire du numéro

Éditeur(s)

Globe, Revue internationale d'études québécoises

ISSN

1481-5869 (imprimé)

1923-8231 (numérique)

Découvrir la revue

Citer cet article

Roy, J. (2004). Des réseaux en convergence. Les espaces de la sociabilité littéraire au féminin dans la première moitié du XIX ${ }^{\mathrm{e}}$ siècle. Globe, 7(1), 79-105. https://doi.org/10.7202/1000831ar
Résumé de l'article

À travers l'étude de quelques correspondances, de certains « salons » et de la pratique de l'album, dans la première moitié du XIX ${ }^{\mathrm{e}}$ siècle, il s'agit d'examiner l'impact de ces espaces de la sociabilité, associés généralement au féminin, sur la formation de la vie littéraire québécoise. Certaines Canadiennes ont investi ces pratiques sociales pour légitimer leurs pratiques littéraires et mobiliser des réseaux d'hommes et de femmes de lettres et d'artistes. Cet article vise donc à ouvrir les perspectives sur les rôles que ces espaces de la sociabilité ont permis aux femmes de jouer dans la sphère lettrée et de comprendre autrement l'émergence de la vie littéraire québécoise. d'utilisation que vous pouvez consulter en ligne. 


\title{
Des réseaux en convergence. Les espaces de la sociabilité littéraire au féminin dans la première moitié du XIX $^{\mathbf{e}}$ siècle
}

\author{
Julie Roy \\ Shadyc, EHESS-CNRS (France)
}

\begin{abstract}
Résumé - À travers l'étude de quelques correspondances, de certains " salons " et de la pratique de l'album, dans la première moitié du XIX siècle, il s'agit d'examiner l'impact de ces espaces de la sociabilité, associés généralement au féminin, sur la formation de la vie littéraire québécoise. Certaines Canadiennes ont investi ces pratiques sociales pour légitimer leurs pratiques littéraires et mobiliser des réseaux d'hommes et de femmes de lettres et d'artistes. Cet article vise donc à ouvrir les perspectives sur les rôles que ces espaces de la sociabilité ont permis aux femmes de jouer dans la sphère lettrée et de comprendre autrement l'émergence de la vie littéraire québécoise.
\end{abstract}

Converging Networks. Women's Literary Sociabilities in 19th Century Québec Abstract - Through the study of various correspondence, certain "salons ", and the making of albums in the first half of the nineteenth century, it is possible to examine the impact of these spaces of sociability, generally associated with the feminine, on the formation of the Quebec literary life. Some Canadian women engaged in these social practices in order to legitimize their literary practices and to mobilize networks of artists and lettered men and women. This article attempts to open new perspectives on the roles that these spaces of sociability allowed women to play in the sphere of letters, and to come to a different understanding of the emergence of the Quebec literary life.

Le chercheur qui tente de comprendre le rôle des femmes dans l'histoire québécoise est sans cesse confronté à des absences, à des lacunes, à des oublis. "Véritable armée de l'ombre, il faut pour arriver à les saisir, user de mille ruses, lire les textes entre les lignes, comparer les chiffres, fouiller les archives et les tiroirs à la recherche de traces habilement

Julie Roy, "Des réseaux en convergence. Les espaces de la sociabilité littéraire au féminin dans la première moitié du $\mathrm{xx}^{e}$ siècle *, Globe. Revue internationale d'études québécoises, vol. 7, n 1, 2004. 
dissimulées ${ }^{1}$. "C'est en effet à partir de l'histoire des hommes que l'on peut bien souvent découvrir l'histoire des femmes, à partir de cette avant-scène constituée de pères, de maris, d'hommes publics, que se dévoile une arrière-scène féminine tout aussi importante. Dans le cas de l'histoire littéraire québécoise, c'est notamment grâce à la reconstitution des réseaux de correspondants que des épistolières et des femmes impliquées dans le monde des lettres finissent par refaire surface ${ }^{2}$. La question institutionnelle du "devenir écrivain " se pose en effet tardivement pour les Canadiens, encore davantage pour les Canadiennes ${ }^{3}$. Les femmes qui privilégiaient une sociabilité partant de la sphère privée et s'inscrivant à la lisière de l'espace public ont eu jusqu'à maintenant peu de chance de voir reconnaitre leur impact. Comme l'indique Allan Greer, au tournant du xIxe siècle, "[s]'afficher en public répugne à une femme honnête parce que c'est là une preuve d'immoralité au même titre que sa réclusion dans l'espace privé est la preuve de sa pudeur ${ }^{4}$. Au Québec, les femmes n'ont généralement pas publié leurs œuvres et n'ont fait partie d'aucun cercle littéraire officiel avant le $x x^{e}$ siècle. En réexaminant les sources, notamment grâce à l'analyse des réseaux, il est désormais possible de les retrouver et de les inscrire dans un cadre d'analyse qui dépasse l'éternel recours à l'anecdotique. C'est sur cet échiquier complexe qu'est le réseau et dans les espaces de sociabilité qui s'érigent aux confins de l'espace privé et de l'espace public que notre compréhension de l'écriture au féminin et de la participation des femmes à la formation de la sphère lettrée québécoise peut sans doute trouver à s'étayer.

1. Marie-Claire Hoock-Demarle, La rage d'écrire. Femmes écrivains en Allemagne de 1790 à 1815, Aix-en-Provence, Alinea, 1990, p. 15.

2. C'est ce que nous avons expérimenté lors de la constitution du corpus de notre thèse, "Stratégies épistolaires et écritures féminines : les Canadiennes à la Conquête des lettres. 1639-1839 ", thèse de doctorat, Département d'études littéraires, Université du Québec à Montréal, Montréal, 2002, 899 f.

3. La première femme qui obtient le label d'écrivain dans l'histoire littéraire québécoise est Laure Conan à la fin du $\mathrm{xIX}^{e}$ siècle.

4. Allan Greer, " La république des hommes. Les Patriotes de 1837 face aux femmes ", Revue d'bistoire de l'Amérique française, vol. 44, nº 4, printemps 1991, p. 513. 
Dans le cas des travaux portant sur le Québec des xvir et $\mathrm{xIX}^{e}$ siècles, plusieurs chercheurs ${ }^{5}$ observent que les cadres traditionnels évacuent un grand nombre de formes et de pratiques en raison de leur inadéquation avec les systèmes de représentation, oblitérant du coup les assises mêmes du littéraire et sa spécificité nationale. Les changements méthodologiques que suscite l'analyse de la sociabilité ouvrent des horizons et permettent d'examiner les rôles que les femmes ont pu jouer dans l'histoire littéraire. En effet, le chercheur pose souvent au passé les questions du présent et les formule à l'intérieur des cadres de sa propre discipline. Or, l'analyse des réseaux et de la sociabilité engage le chercheur à prendre en compte les multiples interdépendances entre les couples d'opposés fondamentaux (homme/femme, politique/domestique, social/intime, professionnel/familial, espace privé/espace public, individu/société, littéraire/non-littéraire) et la nécessaire interaction entre les disciplines. Les frontières deviennent poreuses et il est non seulement possible mais nécessaire de faire interagir hommes et femmes, espace public et espace privé, espace politique et espace domestique, sociologie, histoire et littérature. L'étude des réseaux et de la sociabilité permet d'observer les phénomènes d'interdépendances réciproques ${ }^{6}$, apporte de profonds changements historiographiques en faisant exploser les cadres habituels de l'analyse et de la représentation et offre les outils nécessaires pour analyser et légitimer cette part nébuleuse de l'histoire littéraire québécoise.

Pour comprendre l'importance de la sociabilité, pour illustrer les rôles qu'ont occupés les femmes dans la constitution d'une sphère lettrée au début du xIX ${ }^{e}$ siècle et comment elles ont pu s'y insérer, nous observerons trois lieux de sociabilité généralement associés au féminin, convergeant vers la pratique littéraire, mais souvent laissés en marge des

5. Bernard Andrès et son groupe "Archéologie du littéraire au Québec * (ALAQ) de l'Université du Québec à Montréal travaillent depuis de nombreuses années déjà sur cette question pour le xvII ${ }^{e}$ siècle québécois. Lucie Robert de l'Université du Québec à Montréal et Micheline Cambron de l'Université de Montréal posent également ces questions dans le cadre de leurs travaux respectifs sur l'institutionnalisation du littéraire au $\mathrm{XIX}^{\mathrm{e}}$ siècle.

6. Norbert Elias, Qu'est-ce que la sociologie?, Paris, Pandora, 1981. Ce concept fut repris dans La société des individus, Paris, Fayard, 1990. 


\section{REVUE INTERNATIONALE D'ÉTUDES QUÉBÉCOISES}

analyses de la formation du champ littéraire québécois. Ces trois espaces sont la correspondance (et le réseau épistolaire qu'elle construit et illustre), le salon (emblème de cette sociabilité qui rythme l'existence quotidienne) et l'album (construction réticulaire virtuelle par l'art et la littérature, mais associée à l'espace privé). Si ces trois espaces semblent propices à l'analyse de la sociabilité littéraire au féminin, c'est parce que l'éducation reçue par les jeunes Canadiennes dans les pensionnats et les écoles du tournant du $\mathrm{XIX}^{-}$siècle $^{7}$ visait à faire d'elles de véritables ambassadrices des familles et des relais diplomatiques pour leurs futurs époux. Ces deux rôles entrecroisés devaient permettre leur propre réussite sociale. Or, tout en leur permettant de maintenir les liens sociaux et d'assumer leurs talents pour la conversation, la lettre, le salon et l'album leur donnaient l'occasion de s'initier aux pratiques esthétiques.

\section{L'espace épistolaire}

La correspondance est l'un des matériaux importants pouvant rendre compte aujourd'hui de la constitution des réseaux du passé et de l'existence de lieux de sociabilité. En dehors de sa fonction documentaire, la pratique épistolaire a longtemps été ignorée parce qu'elle se situait en marge des institutions privilégiées par le discours historique. Or, la correspondance est en elle-même un espace privilégié de la sociabilité au tournant du XIX siècle. Hommes et femmes entretiennent des liens avec les membres de leur famille et leurs amis éloignés par le moyen de la pratique épistolaire. La correspondance est le reflet d'un apprentissage à la fois culturel et social nécessitant la maîtrise des règles élémentaires de la sociabilité, mais également celle de l'écriture et de la mise en récit.

7. Il est question ici des écolières des milieux aisés, on en convient, mais ce sont ces femmes qui écrivent des lettres, tiennent salon et participent aux albums, en un mot, peuvent aspirer à devenir des femmes de lettres. Si on en connaît encore peu sur l'éducation fournie aux jeunes filles, on sait toutefois que dans les pensionnats comme dans les classes privées, les institutrices s'efforcent de faire apprendre la lecture, l'écriturè et les règles de la bienséance aux jeunes écolières. Ces apprentissages visaient à leur inculquer les principes de la charité chrétienne par la lecture d'ouvrages pieux, à leur fournir les outils nécessaires à l'entretien de réseaux de correspondances par l'imitation de modèles et à les initier aux règles de la vie mondaine par différents exercices publics. 
Pour la femme en particulier, la pratique épistolaire devient gage de l'intégration de son rôle de gardienne du lien social et la démonstration de sa bonne éducation. Comme le note Cécile Dauphin à propos de l'apprentissage de la lettre par les jeunes filles de bonne famille,

[a]ppliquer et montrer ses capacités à tenir la plume, bien employer son temps en rédigeant et en rendant compte des activités quotidiennes, se conformer au code épistolaire et, à l'occasion, joindre à la lettre un échantillon des ouvrages à l'aiguille ou une fleur séchée, par tous ces gestes la petite fille incorpore la morale du bon usage du temps et les bonnes manières tout à la fois ${ }^{8}$.

La lettre, comme les cadeaux qui l'accompagnent, est avant tout un objet transitoire de l'affection. Si les ouvrages à l'aiguille témoignent des talents de la brodeuse ou de la dentellière, la page griffonnée pour le destinataire peut devenir un espace de création et de diffusion de l'écriture. On offre des nouvelles fraîches, des descriptions, des récits, des prières, des poésies et des chansons, qui s'intègrent à la lettre ou qui s'inscrivent dans sa périphérie et qui témoignent non seulement du naturel des sentiments ainsi exposés, mais également d'un travail bien maîtrisé de l'écriture. Nombreuses sont en effet les femmes qui ont utilisé ce médium pour faire valoir leurs talents littéraires. Plusieurs épistolières s'excusent de la rapidité avec laquelle elles ont dû composer leur missive, témoignant ainsi de leur volonté d'offrir une lettre plus achevée. Quelques-unes expliquent la longueur d'une lettre par leur passion de l'écriture qui rend leur plume incontrôlable et enjoignent le destinataire à la bienveillance. D'autres vont plus loin en insérant dans leur correspondance des essais de littérature. Le 13 février 1809, Marguerite Lacorne joint à l'une de ses missives destinées à son époux, Jacques Viger, le manuscrit d'une chanson qu'elle a composée afin de lui témoigner son affection et d'avoir son avis sur ce premier essai littéraire. Ayant pris connaissance de la pièce, Jacques Viger lui répond le 23 février suivant : "J'ai reçu avec bien du plaisir votre lettre du 13. La chanson que vous

8. Cécile Dauphin, - Questions posées à l'histoire culturelle des femmes. Les manuels épistolaires au xIx siècle ", Genèses, n² 21, décembre 1995, p. 112. 


\section{REVUE INTERNATIONALE D'ÉTUDES QUÉBÉCOISES}

m'envoyez est assurément très jolie et fort de mon goût : les vers en sont bons et de mesure. Merci de la galanterie, et permettez-moi de baiser la jolie main qui me l'adresse". "La réaction de Viger suggère un processus de séduction qui dépasse la mise en scène du sentiment amoureux, la poursuite d'un lien affectif à distance.

Bien que cette chanson s'inscrive dans un espace qui paraît ici strictement privé, penser que les Canadiennes désiraient réellement garder leurs œuvres confidentielles relève d'une vision bien naïve de leur relation à l'écriture et du rôle du réseau épistolaire dans la diffusion du littéraire. En inscrivant cette création dans sa correspondance, Marguerite Lacorne ne fait pas que transmettre de personne à personne une ouvre destinée à divertir son unique destinataire. Elle élargit également son public potentiel. Elle s'inspire d'un genre littéraire que son mari pratique et pour lequel il a acquis une réputation dans son cercle, ce qui lui permet elle-même d'accéder à une certaine forme de reconnaissance. Lettre et sociabilité vont de pair au sens où le papier griffonné, ainsi mis en circulation, peut atteindre d'autres cercles, souvent déterminés, parfois tout à fait inattendus. Dans les correspondances qu'entretiennent les Canadiens, on retrouve fréquemment des recommandations visant à faire lire une lettre, en tout ou en partie, à un tiers. Certaines lettres indiquent que le destinataire a fait naturellement la relecture de la missive reçue à des visiteurs. Ajoutons que les salutations aux familiers qui apparaissent régulièrement en post-scriptum des missives sont sans doute un indice supplémentaire de la lecture des correspondances lors des rencontres mondaines ou, du moins, de la circulation des nouvelles qui y sont inscrites ${ }^{10}$. C'est ici qu'apparaissent les liens entre la lettre et

9. Lettre de Jacques Viger à Marie-Marguerite Lacorne, 23 février 1809, Saberdache, Archives du Séminaire de Québec, vol. 1, p. 148.

10. Elles sont également un moyen de tisser des liens et d'élargir son réseau ou de le consolider. C'est le cas, par exemple, quand Marguerite Lacorne signifie à son mari de ne pas oublier de faire réponse à des personnes qui lui ont été de précieux auxiliaires. Viger, qui s'acquittera ultérieurement de cette tâche, demande à son épouse de faire lire certaines parties des lettres qu'il lui destine aux intéressés et même de leur écrire elle-même. Cette lecture devient un gage de sa reconnaissance et du désir d'inclusion dans ce réseau. Marguerite Lacorne à Jacques Viger, 7 décembre 1808, Saberdache, Archives du Séminaire de Québec, vol. 1, p. 93-97. 
le salon, dont nous parlerons plus loin, et que l'isolement des femmes dans la sphère du for privé peut être brisé.

Les Canadiennes qui s'attablent à leur secrétaire expérimentent et connaissent les possibilités de ce mode de diffusion épistolaire. Au-delà du rôle traditionnel de chroniqueuse, qui répond aux attentes de la société, elles découvrent que l'écriture, qu'elle soit simple chronique ou création littéraire, peut être transmise à un cercle qui va au-delà du destinataire visé et au-delà parfois de la petite société familière ${ }^{11}$. En transmettant leurs compositions par l'intermédiaire de la lettre, les femmes utilisent un médium légitime et s'inscrivent dans une configuration particulière d'échange et de mise en valeur de leur talent. La lettre devient pour elles ce banc d'essai où éprouver les projets littéraires dont parlait Kaufmann ${ }^{12}$, mais également un lieu de diffusion leur permettant d'obtenir une certaine reconnaissance par les pairs. Si les femmes sont conscientes de cette diffusion potentielle, c'est sans doute parce que leur éducation leur a permis d'explorer les possibilités des divers lieux de sociabilité auxquels elles avaient accès. Au couvent, elles apprenaient à composer des compliments qui étaient lus en public lors des fêtes et des examens. Sous le toit familial, c'est au salon, sous le regard attendri des parents et amis qu'elles s'exécutaient. L'exemple de l'éloge de la voix de

11. Par exemple, lors de la fête de mai, célébrée à la seigneurie de Sainte-Mélanie d'Ailleboust en 1839, Eulalie Panet avait présenté quelques libations de son cru en l'honneur des hôtes de la seigneurie. Sa tante, Louise-Amélie Panet, transcrit pour son neveu Guillaume Lévesque, exilé en France, les paroles de la pièce. L'épistolière aurait pu simplement mentionner la tenue de l'exercice. En transcrivant cette chanson, elle diffuse une œuvre au-delà du cercle des invités de sa seigneurie. La transcription des paroles témoigne d'un désir d'inclure le neveu exilé dans la famille, mais également de garder la trace et de diffuser un texte au-delà du cercle familial restreint. Voir Marthe Faribault-Beauregard, La vie aux Illinois. Souvenirs inédits de Marie-Anne Cerré, Montréal, Archiv-Histo, 1987, p. 48.

12. * La pratique épistolaire est indépendamment de son éventuelle valeur esthétique, un passage obligé, un moyen privilégié d'accéder à une œuvre. Et plus généralement, lorsqu'elle ne joue pas ce rôle initiateur, elle fonctionne comme un laboratoire. Elle accompagne le travail de l'écrivain, elle lui permet d'éprouver, dans sa relation à un autre déjà absent, une forme particulière de parole avec laquelle il se tient au plus près de l'écriture proprement dite. "(Vincent Kaufmann, L'équivoque épistolaire, Paris, Minuit, 1990, p. 8.) 
Marguerite Lacorne par le colonel de Salaberry, rapporté par Jacques Viger un mois avant la réception de la chanson de son épouse, montre qu'elle avait l'habitude de ces performances artistiques et qu'elle connaissait les circuits pouvant être empruntés pour trouver un public:

Il me questionna sur votre voix, faisant, en même temps, au Dr Blanchet, les plus grands éloges de votre gosier mélodieux ; \&c, \&c, \&c, On ne peut tarir sur le sujet de l'aimable, de l'inestimable Major. Il m'a rappelé une chanson qu'il vous a entendu chanter si admirablement bien, il m'en a parlé plusieurs fois, et toujours pour me dire qu'il avait rarement entendu une voix comme la vôtre ${ }^{13}$.

C'est donc à nouveau dans le salon familial, où elles savent que leurs lettres et les petits essais de littérature qu'elles contiennent sont lus et relus, que ces productions destinées à la sphère de l'intimité à travers l'échange épistolaire peuvent trouver une audience.

\section{Le salon}

Loin d'être un espace distinct, le salon est la face visible de la sociabilité canadienne, un espace que seuls, en revanche, les témoignages contenus dans les correspondances nous permettent aujourd'hui d'appréhender. On sait que les maisons des administrateurs de la colonie ont été des points de rencontre importants de la bonne société québécoise depuis Frontenac. On en sait toutefois bien peu au sujet des salons privés qui ont sans doute contribué davantage à la vie sociale canadienne. En effet, les descriptions utilisées par les historiens ont été formulées le plus souvent par les invités de ces soirées mondaines, qui étaient pour la plupart des lettrés ou des hommes politiques déjà confirmés. Or, il faut s'interroger sur l'objectif de ces révélations, qui retiennent habituellement les qualités physiques et morales de l'hôtesse ou son affiliation à des individus très en vue dans l'espace public. Rares

13. Jacques Viger à Marie-Marguerite Lacorne, 2 décembre 1808, Saberdache, Archives du Séminaire de Québec, vol. 1, p. 81. 


\section{LES ESPACES DE LA SOCIABILITÉ LITTÉRAIRE AU XIXE SIÈCLE}

sont les hôtes qui entreprennent de décrire les activités qui se déroulèrent dans ces lieux, notamment le rôle précis que ces femmes y ont joué. Par exemple, dans ses Souvenirs, Joseph-Guillaume Barthe écrivait que le salon de Marguerite Lacorne

donnait le ton à notre société d'alors et était le rendezvous de l'élite de notre beau monde où brillait pardessus tous, cette Henriette Nelson, fille adoptive du juge Vallière de St-Réal, devenue plus tard l'épouse du juge Guy. Madame Viger qui en faisait royalement les honneurs n'était rien de moins non plus que mademoiselle de St-Luc Lacorne, veuve du général Lennox, deux de nos plus grandes familles de France et d'Angleterre, dont elle sut soutenir le niveau jusqu'à la fin de ses jours ${ }^{14}$.

Ces femmes qui participent aux activités mondaines sont surtout décrites en fonction des hommes auxquels elles étaient liées et donc des réseaux qu'elles permettaient de tisser. Lorsque Jacques Viger décrit le salon de son amie Louise-Amélie Panet-Berczy, il évoque d'abord l'ambiance agreste de la seigneurie de Sainte-Mélanie d'Ailleboust, ambiance qui attirait des visiteurs venus d'aussi loin que Montréal et Québec. Il offre toutefois peu de détails sur les activités qui avaient lieu lors de ces rencontres et il clôt le poème par la description évasive des quatre maîtresses de maison (Louise-Amélie Panet, Mélanie Panet, Marie-Anne Panet et Jeanne-Charlotte Allamand-Berczy) en donnant à chacune le nom d'une Grâce ou d'une Muse de l'Antiquitée's. Elles ne sont ni les

14. Joseph-Guillaume Barthe, Souvenir d'un demi-siècle ou mémoires pour servir à l'bistoire contemporaine, Montréal, Chapleau et fils, 1885, p. 403.

15. Les deux sœurs de Louise-Amélie (Marie-Anne et Mélanie) sont comparées à Aglaé et Hébé, tandis que Louise-Amélie reçoit le nom de Thalie. Dans la mythologie, ces demi-déesses * concentrent sur la Terre les rayons du soleil, réchauffent le coeur des hommes, embellissent leur vie de jouissances multiples, président aux agréments de la conversation et, plus généralement, aux bonnes relations sociales. Un genre littéraire leur est particulièrement cher, la poésie .. (Schmidt, Dictionnaire de la mythologie grecque et romaine, Paris, Larousse, 1993, p. 52.) Les Précieuses et certaines salonnières avaient également un surnom tiré de la mythologie qu'elles utilisaient pendant les réunions mondaines. Voir Roger Duchêne [éd.], Les précieuses ou Comment l'esprit vint aux femmes, Paris, Fayard, 2001. 
premières ni les dernières Canadiennes à être comparées à ces figures mythologiques alors prisées par les poètes ${ }^{16}$. D'autres mémorialistes utilisent quant à eux l'image de la salonnière d'Ancien Régime, une image toutefois mythifiée à souhait en ce début de xTx siècle. On ne peut toutefois manquer d'observer qu'en faisant des Canadiennes les émules de ces icônes de la République des lettres, les lettrés canadiens se comparaient eux-mêmes aux philosophes des Lumières ${ }^{17}$.

Pour saisir de manière plus réaliste la nature de ces lieux de rencontre et des pratiques qui y étaient exercées, il faut sans doute revenir à des définitions élémentaires et à une vision plus terre-à-terre de ce lieu de sociabilité que constitue le salon. Comme le signale Jürgen Habermas

16. Dans son poème intitulé "L'Heureuse famille *, Joseph-David Mermet dédie quelques vers aux sœurs Quesnel : * À vous admirable Adélaïde/Sœur favorite des neufs sceurs,/Apollon qui toujours vous guide/Doit vous combler de ses faveurs./Et vous charmante Mélanie,/Joyeuse et sensible à la fois,/Vous possédiez l'heureux génie/Dont votre père avait fait choix./Pardonnez-moi si je le nomme,/Quesnel, le père des amours,/Semblable à son bon petit bonhomme,/Vit encore et vivra toujours. "Cité dans John Hare, "Le rôle des salons littéraires à Montréal au tournant du xix siècle ", Bernard Andrès et Marc André Bernier [éd.], Portraits des arts, des lettres et de l'éloquence (1760-1840), SainteFoy (Québec), Presses de l'Université Laval, coll. "La République des Lettres ", 2002 , p. 179.

17. Pierre-Joseph-Olivier Chauveau, invité du salon de Mélanie Quesnel, décrit ainsi l'hôtesse de la rue Saint-Denis : “Mme Cherrier avait beaucoup lu, et les meilleurs auteurs. Elle était familière avec Racine, Molière, Boileau, La Fontaine, leSage et surtout aussi Mme de Sévigné ; elle parłait de cette dernière comme on parle d'une de ses connaissances, de quelqu'un avec qui l'on a vécu. Elle n'en appréciait pas moins les auteurs plus modernes ; elle avait surtout lu avec intérêt les meilleurs mémoires sur l'Histoire de la Révolution et de l'Empire. Elle savait émailler sa conversation de réminiscences littéraires toujours justes, toujours pleines d'à-propos et faites avec si peu de prétention qu'on n'était pas tenté de l'accuser de pédanterie. Elle allait même volontiers jusqu'à la citation latine, mais cela tout en riant et comme en se moquant d'elle-même. Son salon réunissait, chaque dimanche, et assez souvent la semaine, une société peu nombreuse, mais choisie, où l'on causait et l'on s'amusait sans invitation préalable et sans aucune des contraintes de l'étiquette. Qui parmi les habitués, a oublié les agréables soirées du cottage de la rue Saint-Denis ? On l'aurait bien étonnée si on lui avait dit qu'elle faisait précisément elle-même ce qu'elle admirait tant, ce qu'elle racontait si souvent de plusieurs parisiennes dont les mémoires littéraires et politiques des deux derniers siècles nous ont conservé le nom. "Archives nationales du Canada, Fonds Côme-Séraphin Cherrier, MB24 B46.) 
à propos de son importance dans la création de l'espace public au siècle des Lumières,

[l]'espace le plus grand est attribué dans les maisons bourgeoises distinguées à une pièce toute nouvelle : le salon. [...] La ligne qui partage sphère privée et domaine public traverse la maison en son centre même. Les individus quittent l'intimité de leur chambre pour l'espace public du salon. Mais celle-là est strictement corrélative de celui-ci. [...] Les individus qui s'y constituent en public ne se fondent pas au sein de la société ; ils y font en quelque sorte saillie et toujours sur la toile de fond d'une vie privée qui, au sein de l'espace clos formé par la famille restreinte patriarcale, a conquis sa forme institutionnelle ${ }^{18}$.

La sociabilité du salon semble s'apparenter à celle qu'on retrouve dans la définition et dans l'utilisation du terme "société "tel qu'on l'entendait au XvIII siècle : "Société désigne en effet une forme de sociabilité informelle qui dépasse l'opposition entre privé et public, puisqu'il s'agit de l'ouverture de l'espace domestique au-delà du cercle familial ${ }^{19}$. "Dans sa plus simple définition, le salon est donc une pièce de la maison destinée à recevoir des invités. C'est un espace de rencontre, de discussions et d'échanges, un espace mitoyen situé entre l'espace privé où les femmes évoluent et l'espace public dont elles sont généralement tenues à l'écart. C'est un lieu où maintenir les relations sociales, un espace d'aller-retour où les thèmes des discussions peuvent se situer à la fois du côté du privé et du public. Le niveau et les sujets de ces échanges dépendent des personnalités qui fréquentent le salon, du réseau ainsi constitué et de ses centres d'intérêt : aux familiers, les nouvelles familiales ; aux politiciens, la politique ; aux marchands, le commerce ; aux artistes, l'art et aux littéraires, la littérature. Chacun des membres de ces chaînes de relations peut bien entendu être impliqué dans tous ces

18. Jürgen Habermas, L'espace public. Archéologie de la publicité comme dimension constitutive de la sociêté bourgeoise, Paris, Payot, coll. * Critique de la politique $x, 1978$, p. 6.

19. Antoine Lilti, "La sociabilité ", Lucien Bély [éd.], Dictionnaire de l'Ancien Régime, Paris, Presses universitaires de France, 1996, p. 288. 


\section{REVUE INTERNATIONALE D'ÉTUDES QUÉBÉCOISES}

domaines à la fois, comme il peut faire partie du réseau familial, professionnel ou amical d'un individu. Tous les sujets sont donc susceptibles de toucher les membres du cercle, mais certains thèmes reviendront plus fréquemment parmi les invités de certains hôtes en raison de leurs centres d'intérêt et du type de relation qui les unit. Barthe décrivait ainsi les sujets abordés dans le salon de Frédéric-Auguste Quesnel :

Chez lui se réunissait une société d'élite. On commençait dans les salons des joutes littéraires qui se terminaient dans les journaux. MM D. B. Viger, Papineau, Quesnel, J. Viger, Heney, Berczy, jusqu'au vieux baron Schaffelesky, sans être toujours du même avis, savaient se critiquer et s'estimer. La poésie se bornait peut-être trop souvent à la satire et à la chanson politique, mais elle savait aussi parfois prendre un ton tout à fait gracieux. Les deux Dlles Quesnel (Adélaïde et M... (Mélanie]) l'animaient par leurs saillies et les charmes de leur conversation ${ }^{20}$.

Si Barthe témoigne ici du caractère surtout politique du salon d'Auguste Quesnel, il laisse également entendre que les pratiques littéraires y sont relativement courantes. Ces pratiques mènent à des publications dans les journaux, mais elles permettent aussi de soumettre des textes, sans les faire imprimer, à un public d'initiés.

Ce témoignage a certes donné lieu à une certaine valorisation du féminin dans la vie sociale québécoise. Il faut cependant explorer la nature des textes échangés dans cet espace de sociabilité pour éviter de confiner ces pratiques à des images mythiques qui empêchent bien souvent d'analyser leur véritable impact sur la vie sociale et littéraire ${ }^{21}$.

20. Joseph-Guillaume Barthe, Souvenirs d'un demi siècle pour servir à l'bistoire contemporaine, 1885 , p. 404.

21. - Des activités identiques, qui seront considérées comme littéraires dans un réseau littéraire, peuvent très bien devenir des moyens parmi d'autres de produire du politique ou du religieux. De plus nous n'avons pas à attendre que des pratiques soient vraiment littéraires selon nos critères actuels, ou autonomisées pour voir du littéraire. La littérature se fait ni plus ni moins qu'en fonction des critères formels et idéologiques promus par le réseau. " (Manon Brunet, "Prolégomènes à une méthodologie d'analyse des réseaux littéraires. Le cas de la 
Chez Marie-Marguerite Lacorne, la correspondance de son époux Jacques Viger et les débats des gazettes apparaissent comme les pivots des discussions. Dans une lettre du 24 décembre 1808, la maîtresse de maison rend compte des conversations qui ont suivi la lecture d'un article de Jacques Viger paru dans le dernier numéro du Canadien, dont il est rédacteur depuis quelques mois :

Ton adresse aux Canadiens est excellente, elle a été lue avec beaucoup d'intérêt je t'en assure et elle a causé parmi tes amis un plaisir mêlé de surprise car nous nous demandions "Est-ce Jacques qui a écrit cela? Serait-il capable, cet aimable fou (passe nous le mot au moins) d'écrire de cette manière? Si sagement "22.

Quelques années plus tard, Jacques Viger participe à la seconde guerre contre les Américains en tant que capitaine de milice chez les Voltigeurs. Les centres d'intérêt des invités semblent alors être modulés en fonction de ce changement professionnel. Le 23 mai 1813, Marguerite réclame avec insistance le journal promis par son mari :

M. D. B. Viger vous fait ses amitiés et vous prie ainsi que moi de nous donner les détails de la bataille de Saquett car nous sommes bien persuadés que les imprimeurs ne sont pas toujours informés de toute la vérité de ces événements et que la politique préside souvent à la rédaction de ce qu'on en publie \& conséquemment les défigure en partie pour un but et par des motifs jugés bons et avantageux par nos grosses-têtes. Rétablissez donc tout cela en nous disant incessamment la vérité pleine et entière autant que vous en serez capable ${ }^{23}$.

Quatre jours plus tard, Marguerite signale la réception tant attendue du journal et l'appréciation de ses lecteurs :

correspondance de Henri-Raymond Casgrain *, Voix et images, Université du Québec à Montréal, vol. XXVII, $\mathrm{n}^{\circ} 2$ (80), hiver 2002, p. 236.)

22. Marguerite Lacorne à Jacques Viger, 24 décembre 1808, Saberdache, Archives du Séminaire de Québec, vol. 1, p. 105.

23. Marguerite Lacorne à Jacques Viger, 27 mai 1813, Saberdache, Archives du Séminaire de Québec, vol. 2, p. 186-187. 


\section{REVUE INTERNATIONALE D'ÉTUDES QUÉBÉCOISES}

Je vous remercie mille fois du billet que j'y ai trouvé et mille fois encore du plaisir que m'ont donné les détails et les descriptions charmantes de votre voyage. Nos amis à qui je l'ai communiquée m'ont fait les compliments les plus flatteurs, jugez si j'étais fière de me voir si agréablement dédommagée du long silence dont je me plaignais ${ }^{24}$.

Bien que Marguerite Lacorne soit une sorte de relais de l'information et que sa popularité dépende en grande partie de la qualité des renseignements privilégiés auxquels elle a accès, les missives de son mari ne sont pas seulement des substituts des gazettes. Elles sont les prétextes à de nombreuses rencontres et des stimulants à la discussion. D'ailleurs, les lettres de Viger ne sont pas les seuls documents lus et commentés dans son salon. Denis-Benjamin Viger, cousin de Jacques et visiteur assidu de sa belle-sœur, y présente le manuscrit de ses Considérations ${ }^{25}$. Hugh Heney, qui lui rend visite plusieurs fois par semaine, provoquant même la jalousie de son marij ${ }^{26}$, est, tout comme Denis-Benjamin Viger, l'un des journalistes correspondants du Canadien ${ }^{27}$. Les deux hommes s'engageront dans une petite joute oratoire dans le Spectateur de Charles-Bernard Pasteur en 1813, dont on peut aisément situer l'origine dans le salon de Marguerite Lacorne. Si on connaît la prédilection de Jacques Viger pour la chanson et le bon mot, on peut aussi penser, suivant les propos abordés dans sa correspondance avec son épouse, que les extraits littéraires publiés dans Le Canadien ont également suscité des discussions parmi les invités de son épouse, fidèles lecteurs du journal.

24. Ibid., p. 187.

25. Denis-Benjamin Viger, Considérations sur les effets qu'ont produit en Canada, la conservation des établissemens du pays, les mours, l'éducation, etc. de ses babitans et les conséquences qu'entraineroient leur décadence par rapport aux intérêts de la Grande Bretagne, par un Canadien, M. P. P., Montréal, imprimé chez James Brown libraire, $n^{\circ} 20$ rue St-François Xavier, vis-à-vis le Séminaire, 1809.

26. Jacques Viger fait quelques remontrances à sa femme dans une lettre datée du 27 mars 1809 et ajoute quelques commentaires pour l'invité trop assidu, sachant que son épouse lui fait lire ses lettres. (Saberdache, Archives du Séminaire de Québec, vol. 1, p. 178.)

27. Il faut également noter qu'ils deviendront beaux-frères. 
Le baron Schaffaleski, auxiliaire prépondérant de Jacques Viger grâce aux lettres de recommandation qu'il distribue à Québec, visite également Mme Viger à Montréal, alors qu'il loge chez Jeanne-Charlotte Allamand-Berczy. L'épouse du peintre William Berczy, ce très fidèle ami de Viger pendant son séjour à Québec, enseigne l'art et les langues. Une de ses plus brillantes élèves, sa future belle-fille, s'appelle Louise-Amélie Panet. Celle-ci deviendra une salonnière respectée pour ses talents artistiques et littéraires dans les années 1830 et 1840 . En reconstituant les listes d'invités des salons du début du XIX ${ }^{e}$ siècle, on voit apparâtre des espaces où se côtoient des hommes politiques, des journalistes, des amateurs de littérature, des artistes et des femmes d'esprit qui forment un milieu fertile pour le développement de la société canadienne d'alors. Le monde semble d'ailleurs étonnamment petit au Bas-Canada. Les individus qui fréquentent le salon de Marguerite Lacorne depuis 1808 se lient d'amitié avec Louise-Amélie Panet-Berczy dans la décennie 1830 et fréquentent également le salon de Frédéric-Auguste Quesnel, créant ainsi des passerelles entre les disciplines, les arts et les générations ${ }^{28}$.

Ces réunions informelles où la politique, les arts et la littérature constituent des pôles fortement attractifs ne sont peut-être pas encore le signe d'un champ littéraire constitué, mais elles sont sans aucun doute les témoins de lieux où les éléments nécessaires à la réflexion et à la reconnaissance de l'écriture, de l'art et de la littérature se mettent en place. Pierre Rajotte, qui a analysé la question des regroupements intellectuels, précise la formation et le fonctionnement de ces réseaux :

Par définition ce processus présuppose la constitution d'un système d'interrelation dans lequel des individus,

28. Pierre-Georges Roy décrit ainsi le salon de la seigneuresse de d'Ailleboust et la liste de ses invités : “Si madame de Berczy eut continué à fréquenter le grand monde, et se fût manifestée sous son véritable jour, son nom aurait été dans toutes les bouches et son éloge entendu de tous les côtés. La Providence en avait autrement décidé et elle n'a fait les délices que d'un cercle restreint, mais choisi, dans lequel entraient entre autres, MM. Jacques Viger, D. B. Viger, le chevalier d'Estimauville, les deux Stuart, Louis-Joseph Papineau, Henri Heney, etc. Plusieurs de ces hommes distingués correspondaient souvent avec elle, et s'en estimaient heureux. D'autres venaient la voir et ne la quittaient jamais qu'à regret. "(Pierre-Georges Roy, La famille Panet, Lévis, [s. é.] 1906, p. 178.) 
après avoir reçu un enseignement qui les préparait à la réception et, de façon plus limitée, à la production littéraire, sont portés, de par leurs intérêts communs, à se regrouper pour constituer un champ propice à leurs activités, fondés sur l'échange et la reconnaissance entre pairs. Or en se regroupant, ils établissent les frontières qui les spécifient dans le champ intellectuel général et s'affranchissent de la tutelle d'autorités qui prétendent légiférer dans le domaine littéraire en fonction d'un pouvoir extérieur (politique, religion, etc.) ${ }^{29}$.

Marguerite Lacorne réussit à consolider le réseau de son mari pendant ses absences. Elle permet à Jacques Viger de trouver des auxiliaires dans la capitale, dont le major de Salaberry, qu'elle connaît déjà depuis quelques années. Elle réunit, sans peut-être en être consciente, l'un des réseaux qui aura le plus d'influence sur l'évolution de la société québécoise et sur l'émergence de sa littérature dans la décennie 1830. Bien qu'on connaisse surtout ces personnages pour leur implication dans les affaires politiques du pays, plusieurs d'entre eux écrivent régulièrement dans les journaux et s'intéressent aux arts et à la littérature.

Si le salon est un espace de sociabilité majeur, les correspondances témoignent dè ces interrelations et permettent aux individus qui y participent de poursuivre à distance ces moments d'échange et de consolider les solidarités intellectuelles. Dans une lettre que Louise-Amélie Panet envoie au chevalier d'Estimauville pour le remercier de son hospitalité à Québec, elle offre un témoignage des activités qui se déroulèrent lors de leur rencontre :

Les premiers mots que je vous écris doivent être sans doute de remerciements pour la complaisance que vous avez eu de me faire voir quelques-unes de vos productions littéraires. Vos chansons sont très piquantes surtout celle ou vous peignez la clique. D'ailleurs la gaieté avec laquelle vous la chantez triple son mérite. Votre

29. Pierre Rajotte, "Les pratiques associatives et la constitution du champ de production littéraire au Québec (1760-1867)", Revue d'bistoire de l'Amérique française, vol. $45, \mathrm{n}^{\circ} 4$, printemps 1992, p. 569. 
esquisse sur la constitution britannique autant que mes faibles lumières me permettent d'en juger est on ne peut pas plus juste. J'avoue pourtant franchement que je me crois plus en état d'en apprécier le style que la substance. Croyez donc mettant toute espèce de flatterie de côté que je le trouve clair précis et placé et que vous ne pourriez guère en employer de plus analogue au sujet et j'ose ajouter meilleur ${ }^{30}$.

Le reste de la lettre poursuit une discussion amorcée chez le chevalier d'Estimauville au sujet de l'éducation des Canadiens. On peut donc imaginer que la littérature et la réflexion philosophique, telles qu'on les définissait au $x \|_{11}{ }^{e}$ siècle, ont été les ferments des discussions qui se sont déroulées dans le salon de Louise-Amélie Panet, mais que la correspondance a également pu constituer un espace de la sociabilité littéraire canadienne. Pour ces femmes qui avaient difficilement accès à l'espace public, le salon et la lettre ont été des lieux intermédiaires qui ont permis à plusieurs d'entre elles de faire valoir leurs talents et de se créer une réputation de femme d'esprit auprès des lettrés canadiens.

\section{L'album}

L'importance de cette convergence entre le salon et la correspondance apparaît encore plus distinctement lorsqu'on se penche sur cette forme, particulière au xix siècle, qu'est l'album. Avec les échanges de correspondances, la circulation des lettres et des œuvres dans les salons et les performances qui y ont lieu, l'album est' sans doute l'un des moyens les plus propices à la diffusion des talents littéraires et artistiques. Même si on considère que l'album est une pratique qui relève essentiellement de l'espace privé, notamment parce qu'il est le plus souvent du ressort des femmes, il constitue une forme qui se rapproche étonnamment du phénomène de la publication. À partir de la décennie 1830, autant au Québec qu'en Europe, les papetiers diversifient de plus en plus leur production et offrent des papiers fins et des cahiers qui

30. Louise-Amélie Panet à Robert-Anne d'Estimauville, 11 août 1830 , Collection Baby, Archives de l'Université de Montréal, P58, u/9354. 
permettent de multiplier ses usages. C'est à cette époque que l'album devient une pratique en vogue, particulièrement chez les jeunes filles de bonne famille. L'album se définit comme un "cahier destiné à recevoir les productions des écrivains et artistes ${ }^{31}$ ". On y retrouve des textes en prose ou en vers, des partitions de musique, des dessins ou des objets fins d'artisanat ${ }^{32}$. Selon Michelle Perrot, " [c]es pratiques [...] s'inscrivent dans un $\mathrm{Xx}^{\mathrm{e}}$ siècle qui fait du privé le lieu du bonheur immobile, dont la scène est la maison, les acteurs les membres de la famille et les femmes, les témoins et les chroniqueuses ${ }^{33}$ n. En ce sens, les femmes sont perçues non pas comme des collectionneuses qui feraient la promotion de la littérature et des arts, mais comme des "mnémophiles ", c'est-à-dire des collectionneuses de souvenirs. Le nom de celle qui amasse et trie les objets s'efface d'ailleurs bien souvent au profit du plaisir émotif que procure son œuvre ${ }^{34}$. Ainsi, malgré leur étroite parenté, l'album féminin

31. L'encyclopédie du xIx siècle, cité dans Maurice Lemire, La vie littéraire au Québec, tome II, Sainte-Foy (Québec), Presses de l'Université Laval, 1992, p. 271. 32. Des contemporains ont même critiqué ce qu'ils ont qualifié de manie : "Nos demoiselles ont depuis quelques temps une manie, que dis-je une manie, c'est une rage : celle d'avoir un album. Je ne sais d'où leur est arrivé ce nouveau genre; mais ce que je sais fort bien c'est qu'il fait le tourment et le désespoir des jeunes gens de Québec en général et de moi en particulier. "(Le Fantasque, 30 mars 1840.)

33. Michelle Perrot, Les femmes ou les silences de l'Histoire, Paris, Flammarion, 1998 , p. 15.

34. Une lettre de Jacques Viger peut sans doute éclairer cet effacement des femmes. En 1841, il envoie une lettre au chapelain des ursulines afin que soit transmise aux religieuses une lettre adressée en 1699 à Pierre Boucher par sa fille, la mère Geneviève Boucher dite Saint-Pierre (1676-1766). Retrouvée dans les papiers de famille de Marie-Marguerite Lacorne, fille d'une Boucher de Boucherville, cette lettre est offerte aux ursulines de Québec par M. Viger pour les remercier des nombreux documents qui ornent l'album de son épouse. Or, on connaît trois albums de Jacques Viger : celui des communautés religieuses offert au nonce Bedini, celui qui fait partie de la Saberdache et l'album Souvenirs canadiens. Comme collectionneur, Viger vole donc la vedette à son épouse, qui a néanmoins pu participer de près à l'élaboration de ses collections. Si l'album dont parle Viger est bel et bien un album tenu par Marguerite Lacorne, il semble aujourd'hui difficile de le retracer, à moins qu'il n'ait été absorbé par la collection. C'est le cas d'un nombre impressionnant d'albums féminins, dont on mentionne l'existence, mais qui semblent avoir disparu aujourd'hui. (Au sujet de cet échange, voir Histoire des ursulines depuis leur établissement jusqu'à nos jours, tome 2, Québec, C. Darveau, 1878, p. 114-115.) 
et la collection masculine n'ont pas le même prestige ${ }^{35}$. Les hommes auraient recours à la collection en raison de sa capacité à les définir socialement en légitimant leur goût et leur pouvoir pécuniaire, alors que les femmes se situeraient plutôt du côté de la constitution de la mémoire familiale et individuelle ${ }^{36}$.

Il est difficile de vérifier sur le terrain cette apparente dichotomie. En effet, il existe peu d'albums féminins encore disponibles aux chercheurs, peu de témoignages de leur constitution et encore moins d'analyses portant sur cette pratique. Des albums comme ceux de Marie-ReineJosephte Belleau ${ }^{37}$ ou d'Adèle Berthelot-La Fontaine ${ }^{38}$ témoignent d'un art bien maîtrisé, qui est loin d'être aussi dénué d'intérêt pour l'histoire du littéraire que cette division le laisse croire. La constitution d'un album demande un travail de documentation colossal dans lequel les stratégies réticulaires jouent un rôle de premier plan. La première phase de la constitution de l'album, la collecte, est en effet une étape tout aussi signifiante que le résultat esthétique final. C'est celle où l'on sollicite la participation des membres de son cercle, mais également celle de

35. Selon la philosophe Hannah Arendt, " avoir * tiendrait de la sphère privée alors qu'* être * tiendrait de la sphère publique, d'où la distinction qui s'opère généralement entre l'album - ramassis d'objets disparates -, et la collection témoignage du prestige et du bon goût du collectionneur. Voir Hannah Arendt, Condition de l'bomme moderne, Paris, Calmann-Lévy, coll. "Liberté de l'esprit ", 1961.

36. Comme le note Michelle Perrot, "[l]es femmes ont la passion des coffrets, des boîtes et des médaillons où elles enferment leurs trésors : mèches de cheveux, fleurs séchées, bijoux de famille, miniatures qui avant la photo permettent d'emprisonner le visage aimé. Plus tard photos individuelles ou de famille, mises sous cadres ou réunies en albums, ces herbiers du souvenir alimentent une nostalgie indéfiniment déclinée. Recueils de croquis et de cartes postales mémorisent les voyages. Les femmes sont du reste invitées à réaliser de telles collections par l'ingéniosité d'une papeterie en plein essor. *. (Michelle Perrot, Les femmes ou les silences de l'bistoire, Paris, Flammarion, 1998, p. 15.)

37. Marie-Reine-Josephte Gauvreau-Belleau (1811-1884) a confectionné un album conservé dans le Fonds Marie-Reine-Josephte Belleau des Archives nationales du Canada, MG55/24, $\mathrm{n}^{\circ}$ 93. Il contient des dessins, mais également des poèmes de plusieurs lettrés canadiens, dont Augustin-Norbert Morin, ÉtiennePascal Taché, Alfred Garneau et Joseph-Guillaume Barthe.

38. Adèle Berthelot-La Fontaine (?-1859), fille de Joseph-Amable Berthelot et épouse de Louis-Hippolyte La Fontaine, fervente patriote, visite les prisonniers du Pied-du-Courant et y fait circuler son album. 
personnalités extérieures au réseau habituel. Les œuvres offertes, souvent réalisées en fonction d'une demande, acquièrent une valeur importante du fait de leur originalité. Les réseaux de sociabilité et la capacité des femmes à s'y introduire deviennent ainsi d'une importance capitale.

Une lettre de Thomas Chevalier de Lorimier écrite le matin de son exécution à l'intention d'Adèle Berthelot-La Fontaine témoigne d'une requête antérieure adressée au condamné en vue d'obtenir une "relique " du condamné pour son album:

Prison de Montréal, de mon cachot, 15 février 1839

5 heures du matin

Vous me demandez un mot, Madame, pour votre Album. Que puis-je y mettre? Dois-je vous faire du pathétique dans des mots ronflants, du touchant à vous voir fondre en larmes sur mon sort, tandis que ma situation sans les écrits et les paroles vous montrent le comble du malheur dans ma personne infortunée, vous assurez de mon respect? Lorsque toute ma conduite passée a été pour vous le témoigner dans chaque circonstance [sic]. Malgré tout, en définitive, je croirais manquer à mes devoirs envers vous, Madame si je ne me soumettais pas ne souscrivais pas [sic] à votre désir. Permettez-moi donc de vous prier de penser à moi, vous me survivrez, je vais périr sur le gibet politique dans quatre heures, mais faites que je vive dans votre estime et celle de votre époux, Mr Lafontaine. Cette idée me supportera dans mes derniers instants, jointe à celle de la cause sacrée pour laquelle je vais bientôt expirer. Adieu madame, soyez heureuse ainsi que Mr votre époux, vous le méritez. C'est le vou d'un homme qui va dans un instant monter sur l'échafaud pour son pays et sa liberté. Adieu.

Chère dame Adieu

$\mathrm{CDL}^{39}$

39. Album Adèle Berthelot-La Fontaine, Archives du Musée Stewart, Île SainteHélène, Montréal. 
On connaît mieux cet album grâce aux nombreux témoignages de prisonniers politiques qu'il contient. Mme La Fontaine visitait régulièrement les détenus à la prison du Pied-du-Courant et son album circulait parmi les prisonniers, qui trompaient l'ennui en y inscrivant quelques mots ou un dessin pour celle qui leur apportait un peu de réconfort ${ }^{40}$. Contrairement à l'image de l'album féminin, généralement nourri de l'intimité familiale, celui de Mme La Fontaine possède avant tout une valeur pour l'histoire politique du pays, d'où peut-être sa conservation. Le choix des participants et le lieu dans lequel ils s'inscrivent en témoignent et permettent de reconstituer un réseau, celui des patriotes, mais également celui des lettrés canadiens qui laisseront leur marque pendant la décennie subséquente. Adèle Berthelot n'est pas la seule à utiliser ses relations pour enrichir sa collection. Un mois après l'exécution de de Lorimier, Odile Cherrier s'adresse au baron de Fratelin afin d'obtenir le portrait du condamné qu'il avait réalisé à la prison de Montréal quelques heures avant le moment fatidique. Henriette Cadieux de Lorimier l'avait réclamé au baron au lendemain même de la mise à mort de son mari. Tandis que pour Mme de Lorimier le portrait de son époux est un souvenir bien personnel, pour Odile Cherrier il semble déjà posséder une valeur historique ${ }^{41}$.

Si l'album est généralement destiné à un usage privé, il se définit aussi socialement par rapport au public qui aura accès au collage. La femme qui crée l'album s'inscrit en filigrane comme organisatrice de l'information ; sa présence apparaît à toutes les étapes de la constitution,

40. On y retrouve des cuvres littéraires et picturales de Louis-Hyppolite La Fontaine - son mari -, de Joseph-Guillaume Barthe, de George Boucher de Boucherville, d'Augustin-Norbert Morin, de Robert Shore Milnes Bouchette, d'Henri Cartier, de Jean-Philippe Boucher Belleville, de Ludger Duvernay et de Pierre-Joseph-Olivier Chauveau.

41. Odile Cherrier explique au baron : "Il est inutile que je vous parle de ma douleur, votre cœur sensible et généreux doit sans doute vous dire ce que le mien souffre après une semblable perte. Il n'y a qu'une chose qui me console dans ma peine. Souffrez que je vous le dise, c'est la haine que je porte au nom Anglais, et j'espère que plus d'une âme dans la prison surtout sympathise avec moi sur ce point. C'est aujourd'hui l'anniversaire du jour où la pauvre victime a consommé son sacrifice. Ah! Si c'était du moins un jour de vengeance et de représailles !... Pardon, je vais peut-être un peu loin. " (15 mars 1839, Fonds événements 1837-1838, Archives nationales du Québec à Québec.) 
témoignant de son goût pour l'art et pour la littérature et de son intégration au réseau qui s'érige au fil des pages ${ }^{42}$. D'abord, elle choisit et préserve les objets qui feront partie de l'album. Ce tri permet ensuite de classer les objets selon une visée esthétique qui doit prendre en compte la présentation de l'album à un cercle d'amateurs. Ainsi, l'album n'est pas un simple collage aléatoire de productions artistiques et littéraires, mais il devient une forme de représentation esthétique de soi. Il permet à des jeunes filles et à des femmes de faire œuvre de création en assumant un rôle de conservatrice au sens quasi muséologique du terme. Elles choisissent et disposent des œuvres en fonction de critères esthétiques qui les mettront en valeur sur la scène artistique et littéraire et qui leur conférera un rôle de mécène dans leur réseau. Les collaborateurs, qui font généralement de la collectionneuse leur muse, accentuent cette fonction de l'album. Le réseau qu'elles utilisent et qu'elles construisent à la fois s'y reflète et devient une véritable carte de visite. Malgré leur inscription dans la sphère privée, ces albums ont permis de rassembler des œuvres, de les faire circuler dans les réseaux créés par les collectionneuses et de nous les rendre encore accessibles aujourd'hui.

Comme pour la correspondance et pour le salon, l'avantage de l'album aux yeux de celles qui s'y consacrent réside dans le caractère privé de sa pratique. Même si sa finalité est d'atteindre un public, il ne se destine pas à l'espace public. La distinction est subtile, mais elle joue un rôle non négligeable dans la prise d'assaut de cé genre par les femmes et dans l'insertion de certaines ouvres féminines dans les pages des albums. L'album Souvenirs canadiens de Jacques Viger permet de saisir les enjeux de ce lieu de consécration pour les Canadiennes ${ }^{43}$. Dans le domaine artistique, on retrouve des portraits, des paysages, des planches

42. On rapproche le genre de l'album de ce que les Américains appellent le * scrapbook * et qui a donné lieu à l'émergence d'un vocabulaire typique de cette pratique appelée le "scrapbooking - et à une industrie de papeterie prolifique. Selon Tamar Katriel et Thomas Farrell, le "scrapbook " ou "l'album de découpures " est un art de la mémoire, du collage et il peut être perçu comme une construction de l'identité au sens métonymique. Tamar Katriel et Thomas Farrell, "Scrapbooks as Cultural Texts: An American Art of Memory? ", Text and Performance Quarterly, vol. 11, $\mathrm{n}^{\circ} 1,1991$, p. 1-17.

43. Viger insère une série de portraits de femmes illustres de la Nouvelle-France, réalisés par James Duncan. 
représentant des éléments de la faune et de la flore à la manière des herbiers ornés d'illustrations ainsi que des ouvrages de fantaisie. Parmi les Canadiennes identifiées, Julia Mary Cuthbert ${ }^{44}$ offre quelques aquarelles et trois poèmes ("Scrap Book ", "Poème anglais " et "The Arab to His Head .), tandis que Louise-Amélie Panet présente une description poétique de l'été des Sauvages ${ }^{45}$ et un paysage au crayon et à l'aquarelle intitulé "Le combat de la Grange ". L'album répond aux attentes formulées par plusieurs littérateurs canadiens qui souhaitent que les femmes s'initient à la littérature, mais qu'elles préfèrent les joies de la lecture et les émotions contenues dans la sphère privée au vain sentiment de triomphe que produit la diffusion d'une œuvre au grand public.

Si l'album convient parfaitement aux jeunes filles qui aiment la littérature et l'art sans pour autant prétendre à une carrière d'écrivain ou d'artiste, il permet aussi à des Canadiennes de participer à l'espace artistique et littéraire et d'obtenir une certaine légitimation. En effet, le confinement de l'album au for privé est tout aussi illusoire que le cantonnement de la lettre féminine à l'espace intime. L'album circule et les commentaires des collaborateurs montrent bien qu'il constitue à leurs yeux un espace de consécration et le lieu possible d'une reconnaissance par les pairs. On sait par exemple que Jacques Viger présentait son album Souvenirs canadiens à des visiteurs de passage ; quelques-uns y laissaient leur signature, d'autres un écrit ou un dessin. Viger faisait également circuler son album parmi ses compatriotes. Une lettre de Pierre-Joseph-Olivier Chauveau en témoigne. Au feuillet 643 , on trouve, daté du 27 juin 1856, ce commentaire : "En vous renvoyant votre album si riche [...] permettez-moi d'ajouter quelques vers ${ }^{46}$. En ce sens, l'album s'apparente à la lettre. Il s'inscrit d'abord dans la sphère privée, où il s'adresse à un réseau circonscrit et choisi, mais il circule aussi et permet ainsi la diffusion des œuvres ${ }^{47}$.

44. Nièce par alliance de Louise-Amélie Panet.

45. Le poème "Description de l'été de la Saint-Martin 1838 * est un extrait du poème " Quelques traits particuliers aux saisons du Bas-Canada et à l'habitant de ses campagnes mis en vers . Ce poème a été édité par Roger Le Moine, Ottawa, Éditions David, 1999.

46. Album Souvenirs canadiens, Bibliothèque de la Ville de Montréal.

47. Jacques Viger finira même par offrir sa riche Saberdache, autre collection imposante, à Michel Bibaud afin qu'il y puise des textes à imprimer dans $L a$ 
Les femmes qui choisissent de participer aux albums sont conscientes de cette fonction de légitimation et de diffusion. À l'été 1841, Louise-Amélie Panet écrit quelques vers pour l'album de Marianne Stuart, fille de l'un de ses invités ${ }^{48}$. Dans ce poème, composé de six strophes, elle suggère à Mlle Stuart de ne pas s'enflammer vainement à la vue des honneurs et elle lui conseille de préférer un mode de vie où la modestie et l'effacement sont gages de tranquillité. Pour cette femme qui écrit régulièrement des œuvres en vers, mais qui aurait refusé de les laisser publier dans les journaux ${ }^{49}$, l'album est un espace de diffusion, tout comme l'étaient la lettre et le salon. La poétesse note en post-scriptum une petite inconvenance dans la métrique du poème : "Il ne faut pas, dit-on, mettre trois vers féminins de suite dans les stances. Je connaissais cette règle. Mais je trouve mes productions de si peu de conséquence que mes pensées s'étant formulées de cette manière, je les ai laissées prendre leur cours ${ }^{50}$. Cette remarque montre que Louise-Amélie Panet est consciente de la possible diffusion de son cuvre, ce que confirme la thématique de la modestie féminine qui apparaît dans son poème. Il lui faut respecter les idées reçues en matière de féminité pour conserver l'estime de ses pairs et construire sa crédibilité selon les règles du jeu de la reconnaissance. L'album apparaît ainsi comme un espace de prédilection pour les femmes qui souhaitent diffuser leurs œuvres et s'inscrire

Bibliothèque canadienne. Confiant dans la valeur de l'œuvre soumise à l'examen de son collègue, Viger explique : "Quelle mine à exploiter que cette Saberdache pour votre Bibliothèque canadienne! Vous y trouverez d'un côté, des vers... bons quoiqu'ils ne soient pas de moi; de la prose... comme je n'en écrirai pas de meilleure ; bref une correspondance inédite entre Canadiens et Européens. " (Jacques Viger à Michel Bibaud, Saberdache, Archives du Séminaire de Québec, vol. VII, p. 215-217.)

48. Louise-Amélie Panet, "Si tout votre avenir se fonde", Collection Baby, Archives de l'Université de Montréal, P58, boîte 95, Pièces diverses, Q1/162.

49. * Avec son imagination vive et d'une grande abondance d'idées, il était naturel qu'elle aimât la poésie, aussi elle s'y livrait avec plaisir, et dans ses moments de loisir, elle produisit plusieurs pièces de sa composition, fort marquantes ; mais son abnégation d'elle-même et l'opinion modeste qu'elle entretenait de ses productions littéraires, l'empêchèrent de les mettre au jour et lui firent refuser même à quelques-unes de ses connaissances qui l'en avaient sollicitées d'en laisser publier. " (William Bent Berczy, cité dans Marthe Faribault-Beauregard, La vie aux Illinois, Montréal, Archiv-Histo, 1987, p. 30.)

50. Louise-Amélie Panet, "Le Houipp-eur-ouil ", Collection Baby, Archives de l'Université de Montréal, P58, boîte 95, Pièces diverses, Q1/162. 
dans des réseaux de lettrés qui puissent leur apporter une certaine légitimité. Quant à celles qui tiennent un album, ce dernier leur permet de jouer un rôle de pivot dans la création de réseaux littéraires et de mettre en scène leur autorité dans la sphère lettrée.

Les espaces de la sociabilité, loin d'être confinés au for privé, sont des lieux frontières. C'est pourquoi les cadres d'analyse habituels en font de véritables apories pour le discours historique. Comme l'indique Pierre Rajotte,

[t]out différents qu'ils soient, ces modes de socialisation illustrent, chacun à sa façon, comment les agents du champ littéraire entrent en interaction pour en arriver à se former et à se reconnaître mutuellement et, partant, à conquérir une autonomie et une légitimité proprement littéraire ${ }^{51}$.

Ces espaces de la sociabilité que sont les correspondances, les salons et les albums mettent en lumière des dynamiques sociales dont les femmes sont parties intégrantes. Ils se présentent aussi, et surtout, comme des lieux où hommes et femmes, de concert, ont façonné l'espace culturel québécois. Si les femmes se sont rarement inscrites dans l'espace public, elles n'en ont pas moins joué un rôle important dans les différents réseaux auxquels elles se sont intégrées et qu'elles ont souvent contribué à former. Le confinement des femmes à ces espaces en marge des instances de consécration (un confinement qu'elles ont assumé et parfois même revendiqué) peut sembler un paradoxe lorsqu'on considère les indices qu'elles ont laissés de leur désir d'obtenir la reconnaissance des pairs et les stratégies qu'elles ont utilisées pour y parvenir. Le paradoxe n'est qu'apparent. Si les femmes redoutent la publication, c'est que la diffusion publique entraîne avec elle une perte de contrôle de l'œuvre, qui se trouve ainsi exposée à la critique. Bien que les hommes soient tout aussi vulnérables (les polémiques qui apparaissent dans la presse à la même époque peuvent facilement nous en convaincre), les femmes ont le désavantage d'être directement associées à leur œuvre : c'est non

51. Pierre Rajotte [éd.], Lieux et réseaux de sociabilité littéraire au Québec, Québec, Nota bene, 2001, p. 21. 
seulement leur production qui sera attaquée, mais leur identité de femme. C'est donc dans les espaces de la sociabilité où elles ont appris à exceller, dans ces lieux de circulation limitée à un système d'interconnaissance, qu'elles peuvent donner libre cours à leurs talents littéraires. Aussi peuvent-elles tenir un rôle non négligeable dans la formation de ces espaces de la sociabilité, qui sont au cœur de la formation du champ littéraire québécois dans la première moitié du XIX ${ }^{e}$ siècle.

Il est évident que l'analyse des réseaux et de la sociabilité propres aux correspondances, aux salons et aux albums ne permet pas une cartographie exacte des interrelations qui ont contribué à la formation du champ littéraire. Pareille analyse implique qu'on se fie à des sources qui, bien qu'elles apportent souvent de nouvelles réponses, n'en demeurent pas moins lacunaires. En effet, les traces écrites qui subsistent dépendent des choix des conservateurs, mais également des desseins particuliers des rédacteurs. Une correspondance est le reflet d'un pacte épistolaire entre deux personnes et les indices permettant de saisir les chaînes d'interrelations d'un individu ne sont souvent que ceux qui intéressent l'unité formée par l'épistolier et le destinataire. Il faut également opérer une distinction entre réseau potentiel et réseau activé lorsqu'il s'agit de traiter les chaînes d'interrelations. Plusieurs individus peuvent apparaître dans un espace dé sociabilité et ne pas entretenir de relations privilégiées avec cet espace, tandis qu'un individu sans appartenance officielle au réseau peut y jouer un rôle d'importance. L'analyse du rôle des femmes dans les réseaux de sociabilité littéraire se heurte à ces deux difficultés. Ce sont là des éléments dont on doit tenir compte, mais qui n'empêchent pas d'entrevoir, à long terme, une transformation de la façon d'aborder l'histoire littéraire.

L'analyse réticulaire et l'étude de la sociabilité découlent des avancées notoires produites par la multiplication des travaux dans le domaine de la sociologie. Elle est également le propre des transformations récentes de l'historiographie, qui a commencé à s'intéresser à l'histoire sociale et à l'histoire des femmes et à varier les sources utilisées. La valorisation des écrits personnels, en particulier de la pratique épistolaire, comme objets d'étude et d'analyse y ont également contribué. Les correspondances sont des matériaux qui permettent de rendre compte 
de la constitution des réseaux et de l'existence de lieux de sociabilité. Les correspondances féminines, longtemps négligées en raison de leur association à la sphère privée, trouvent dans cette perspective tout leur intérêt. Les quelques exemples présentés ici ont été choisis parce qu'ils ont l'avantage de s'inscrire dans un réseau plus ou moins circonscrit composé de personnages connus. Loin de constituer des hapax, ces femmes impliquées dans des réseaux de sociabilité comme épistolières, comme maîtresses de maison ou comme conceptrices d'albums sont plus nombreuses qu'on ne le croît au sein de la bonne société canadienne. C'est par une lecture attentive des correspondances et par la reconstitution des réseaux de sociabilité que l'on pourra découvrir des aspects négligés de l'histoire culturelle québécoise, plus spécifiquement en ce qui a trait à la part que prirent les femmes à cette histoire. Puisse cette ébauche d'un terrain d'analyse riche et complexe servir d'amorce à une réflexion d'ensemble sur l'importance des espaces de la sociabilité littéraire dans l'histoire littéraire québécoise, une histoire où espace privé et espace public se sont mutuellement façonnés, où hommes et femmes ont eu un rôle à jouer. 